\title{
The Determinants of Business Analytics Adoption: Does One-Size Fit All? ${ }^{1}$
}

\author{
Nazlı BAYRAM iD a Melek AKIN ATEŞ ${ }^{\text {iD }}$ b \\ a Central Bank of the Republic of Turkey, Ankara, Turkey. nazli.bayram@tcmb.gov.tr \\ b Sabanci University, Sabanci Business School, Istanbul, Turkey. melek.ates@sabanciuniv.edu
}

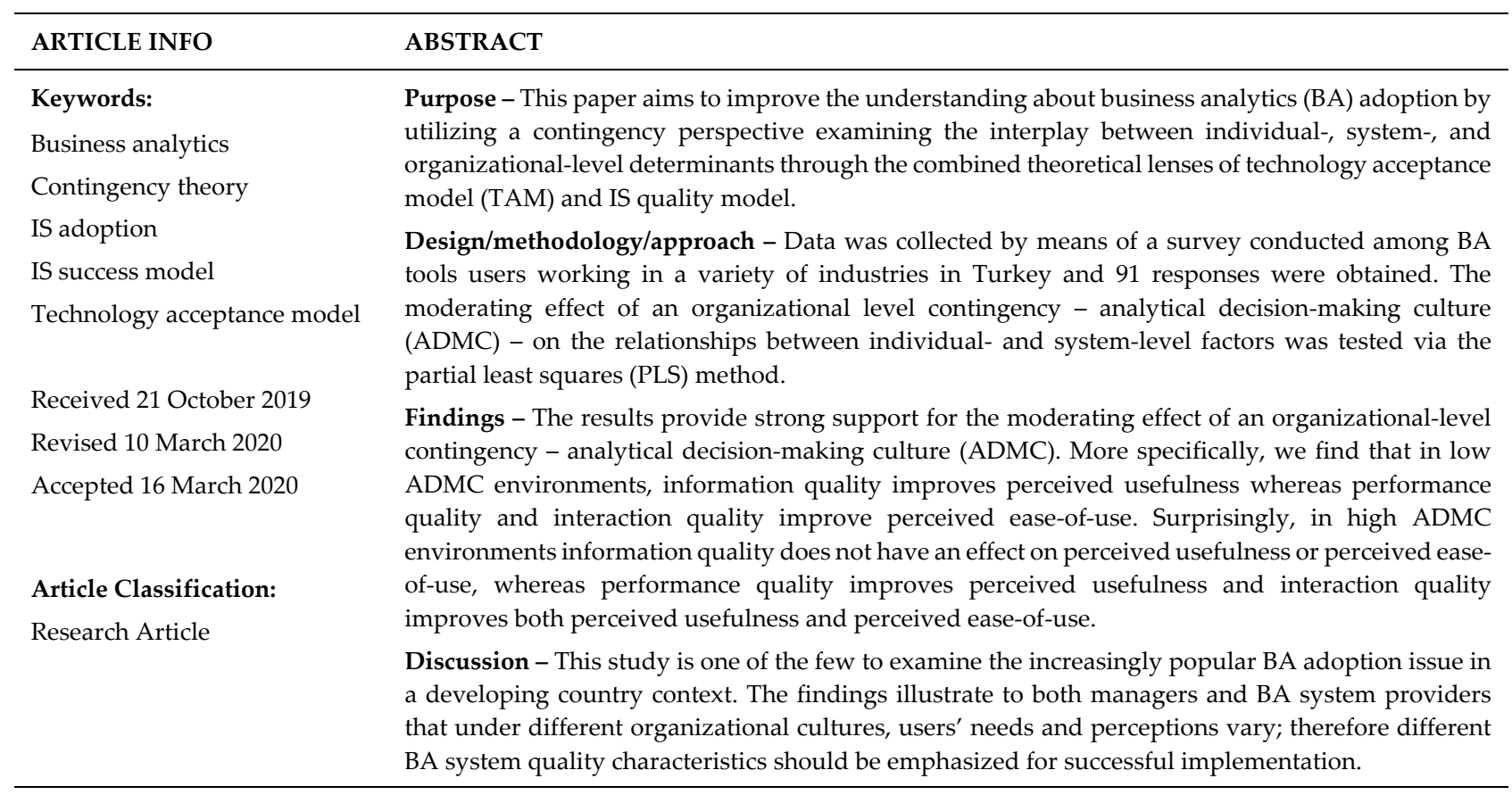

\section{Introduction}

With the growth in international trade supported by digitalization in the past decade, firms are undeniably being immersed with massive amounts of structured and unstructured data (i.e., big data) flowing in from all parts of their operations (Delen and Ram, 2018; Nam et al., 2019; Popovič et al., 2018). While creating a lot of opportunities for firms, big data also makes it necessary to adopt systematic business analytics approaches (Cao and Duan, 2017; Côrte-Real et al., 2019; Popovič et al., 2018). Business analytics (BA) can be defined as "the extensive use of data, statistical and quantitative analysis, and explanatory and predictive models" (Davenport and Harris, 2007, p.7). Big data enables better organizational decision-making and problem solving (Delen and Ram, 2018) and generates competitive advantage (Raguseo and Vitari, 2018; Trieu, 2017). The 2019 New Vantage Partners Big Data and AI Executive Survey report that 55\% of the participating Fortune1000 firms have BA investments in big data exceeding $\$ 50 \mathrm{M}$ (Davenport and Bean, 2019). Therefore, it is crucial for firms to generate the maximum value out of these costly BA investments (Côrte-Real et al., 2019; Nam et al., 2019; Raguseo and Vitari, 2018).

In line with the growing popularity of BA, there have been academic and practical interest in understanding the potential outcomes of BA (Davenport and Bean, 2019; Cao and Duan, 2017; Delen and Ram, 2018). While studies have found that BA can help firms formulate and implement better strategies, improve customer satisfaction, and generate competitive advantage (Bach et al., 2016; Cao and Duan, 2017; Kiron et al., 2014; Raguseo and Vitari, 2018; Trieu, 2017), others have found that not all firms benefit from BA to the same extent. A strong determinant of BA success is enterprise-wide adoption of the BA tools by all employees throughout the organization (Jaklič et al., 2018; Lin and Lin, 2019). Because the use of BA tools is often optional (Popovič

${ }^{1}$ This article is based on the master thesis of Nazlı Bayram (Middle East Technical University, Graduate School of Social Sciences, 2018). 


\section{N. Bayram - M. Akın Ateş 12/1 (2020) 583-598}

et al., 2012), employees' behavioral intentions to use BA is of vital importance. The obtrusive behaviors of employees related to resistance towards using BA tools might even reduce firm performance (Jaklič et al., 2018; Verma et al., 2018). Considering this phenomenon, in this study we examine the factors affecting BA use of employees by means of an integrative model and adopting a contingency perspective.

There are two widely adopted models in the literature that explain information systems (IS) use. First, Technology Acceptance Model (TAM) (Davis, 1989) focuses on the individual-level antecedents, and proposes that (i) the perceived ease of use (PEOU) and (ii) the perceived usefulness (PU) are the two main determinants of individual's attitude and intention to use IS. TAM has been widely used in the literature to examine technology adoption as it is regarded as parsimonious, verifiable, and applicable to a variety of settings (Rauniar et al., 2014). Second, the IS success model (DeLone and McLean, 2003) focuses on the system-level determinants, and proposes that (i) information quality and (ii) system quality are associated with IS adoption. These two models have been vastly examined in relation to various IS contexts such as ERP systems, eprocurement tools, internet banking and e-government services (Alomari et al., 2012; Brandon-Jones and Kauppi, 2018; Lee et al., 2010).

However, we do not know how individual-level and system-level antecedents together play a role in IS use, especially in the context of new IS technologies, such as BA. The little extant work on BA adoption (Cao and Duan, 2017; Daradkeh, 2018; Verma et al., 2018), albeit very informative, do not address this research gap. In order to build up a comprehensive model of BA use, we develop a conceptual model that integrates the TAM and IS success models. An integrative model should also consider the organizational-level contextual factors, because BA adoption would unfold in idiosyncratic ways in different organizations. We propose that the analytical decision-making culture (ADMC) of the organization, the extent to which a firm encourages decision makers to systematically use data and facts and analyze them for their decision-making tasks (Kulkarni et al., 2017; Popovič et al., 2012), moderates the relationships between the individual-level and system-level determinants and the BA use intentions. As opposed to the commonly held view that a high ADMC environment is more fruitful for BA implementation (Božič and Dimovski, 2019; Trieu, 2017), in this study we propose that in both low vs. high ADMC environments BA can foster; albeit being encouraged by different determinants, hence adopting a contingency perspective.

In sum, we aim to answer the following research questions:

RQ1: How are the individual- and system-level determinants of business analytics use intentions related?

RQ2: How does analytical decision-making culture affect the relationship between individual- and system-level determinants as well as their impact on business analytics use intentions?

This study makes several contributions to the literature. First, we offer a holistic theoretical model of BA use intentions that brings together the TAM and IS success models. This model would equip the scholars in both fields with a conceptual lens to juxtapose the hitherto distinct but related literature streams. Second, our results suggest that the impact of IS quality dimensions on key TAM variables, PEOU and PU, is contingent on the ADMC environment, thus proposing a possible explanation with respect to conflicting findings in earlier studies. More specifically, we find that in firms with a low ADMC, information quality has a significant positive effect on $\mathrm{PU}$, and performance quality and interaction quality are only associated with higher levels of PEOU. However, in firms with a high ADMC, information quality has no effect on PEOU or PU, and performance quality and interaction quality are associated with higher levels of PU (as opposed to PEOU in low ADMC environments). Furthermore, in high ADMC environments, only interaction quality is associated with PEOU. These findings hold important scholarly and practical implications. Third, we validate TAM in a rather less examined context: BA system use in a developing country. We illustrate that the model has high predictive validity in both low and high ADMC environments; the main relationships between TAM variables hold irrespective of the ADMC environment.

Our study also provides instrumental insights for both managers and BA system providers. Although TAM has been found to successfully explain cognitive factors affecting individuals' IS use intentions, it is limited in terms of managerial guidance regarding which specific actions to take (Brandon-Jones and Kauppi, 2018). By distinguishing between low and high ADMC environments and illustrating varying effects of different IS quality dimensions, we illustrate to decision makers that a one-size-fits-all approach does not work. 


\section{N. Bayram - M. Akın Ateş 12/1 (2020) 583-598}

The rest of the paper is structured as follows. First, we provide the theoretical background discussing the importance of business analytics, IS success model, TAM model, and the contingency role of ADMC. Then, we present the hypotheses illustrating our conceptual model. In the Research Methods section, we discuss questionnaire development as well as the sample and data collection efforts. In the Results section, we illustrate the findings obtained via PLS-SEM method. Finally, in the Conclusion section we elaborate on our findings by discussing theoretical and managerial implications, stating limitations, and making suggestions for future research.

\section{Theoretical Development}

\subsection{Business Analytics}

Business analytics (BA) is defined as the art and science of discovering insights by using sophisticated mathematical, statistical, machine learning, and network science methods (Cao and Duan, 2017; Delen and Ram, 2018). Although several related terms such as decision support systems and business intelligence have been used in the literature, more recently these terms have slowly been replaced by BA due to the increasing emphasis on big data applications (Cao and Duan, 2017; Delen and Ram, 2018; Nam et al., 2019). Increasing volume, variety, and velocity of data necessitate using descriptive, predictive, or prescriptive analytical methods for translating raw data into business insights (Popovič et al., 2018; Verma et al., 2018).

Recent studies find that with investments in BA systems, firm are able to make more accurate demand forecasts, improve product development, and coordinate supply chain planning more effectively (Božič and Dimovski, 2019; Puklavec et al., 2018; Trieu, 2017). Ultimately, these improvements increase customer satisfaction, operational performance, financial performance, and generate a competitive advantage (Cao and Duan, 2017, Kiron et al., 2014; Ragueso and Vitari, 2018). However, studies also argue that in several cases large BA investments fail and do not always bring the expected outcomes (Nam et al., 2019; Yeoh and Popovič, 2016). One possible explanation to this is the unsatisfactory acceptance rates of users (Popovič et al., 2012).

BA can be used by both senior managerial levels for strategic decision making, but also at lower managerial levels for improving efficiency in performing daily tactical and operational tasks (Arnott et al., 2017). In that sense, it is important to examine factors that increase the motivation of employees to use BA systems. Although IS literature has examined user adoption of several IS technologies, there have been few studies that explicitly focus on BA systems (e.g. Daradkeh, 2018; Nam et al., 2019; Verma et al., 2018). In order to identify the determinants of BA system use, in this study we focus on Technology Acceptance Model (TAM) and IS success model, explained in detail in the next sub-sections.

\subsection{Technology Acceptance Model (TAM)}

TAM, proposed by Davis (1989), is an adaptation of the theory of planned behavior which proposes that the intention to perform a specific behavior depends on the attitude toward that behavior (Fishbein and Ajzen, 1975). TAM has been widely adopted to explain user acceptance of a technology (Alomari et al., 2012; Hong et al., 2013). It has been validated in several contexts such as ERP systems (e.g. Alhirz and Sajaev, 2015); eprocurement tools (e.g. Brandon-Jones and Kauppi, 2018); technologies in life-insurance industry (e.g. Naicker and van der Merwe, 2017) and e-government services (e.g. Alomari et al., 2012). Although different versions of traditional TAM have been proposed in the literature (e.g. TAM2, UTAUT), in this study we adopt the traditional TAM, due to its parsimony and significant explanatory power (Gangwar et al., 2015; Hong et al., 2013). Furthermore, it has an inherent link to the IS success model (Verma et al., 2018) which we build on to identify system-level determinants of BA use intentions.

TAM is a cognitive framework proposing two key determinants of behavioral responses: perceived ease of use and perceived usefulness (Davis, 1989). Perceived ease of use (PEOU) is defined as the degree to which an individual believes that using a particular system would be free of effort (Verma et al., 2018), whereas perceived usefulness (PU) refers to the degree to which an individual believes that using a particular system would enhance job performance (Davis, 1989). PEOU relates more to intrinsic motivations such as enabling flexibility, ease, and comfort for the user, whereas PU relates more to extrinsic motivations such as generating valuable system outcomes such as efficiency, effectiveness and enhanced job performance (Gangwar et al., 2015; Lin and Lin, 2019). One of the main premises of TAM is that the external determinants do not have a direct effect on attitude or behavior, but they affect indirectly through PU and PEOU (Davis, 1989). 


\section{N. Bayram - M. Akın Ateş 12/1 (2020) 583-598}

While TAM enables us to understand the cognitive mechanism for transforming IS value to actual use, it falls short of explaining what generates such value in the first place (Brandon-Jones and Kauppi, 2018). In this study, we rely on IS success model of DeLone and McLean (1992) to identify the external determinants of PEOU and PU (Verma et al., 2018).

\subsection{IS Success Model}

IS success theory (DeLone and McLean, 1992; Wixom and Todd, 2005) is widely adopted to examine IS quality outcomes both at the organizational-level such as business value, efficiency and product/service enhancement (Gorla et al.,2010; Ji-Fan Ren et al., 2017), and also at the individual-level such as beliefs in the benefits, engagements, and usage (Peters et al., 2016; Verma et al., 2018). In this study, we focus on the individual-level outcome of intention to use, and distinguish between two key dimensions of IS quality characteristics that impact users' value perceptions: information quality and system quality (DeLone and McLean, 1992; Wixom and Todd, 2005).

Information quality (INFQ) is defined as the user's perception of the output quality generated by an IT system (DeLone and McLean, 1992) and is characterized by factors such as accuracy, completeness, reliability and timeliness. (Gorla et al., 2010; Wixom and Todd, 2005; Verma et al., 2018). One of the first outcomes users expect from BA systems is INFQ allowing to find patterns in data and translate them into useful information (Ji-fen Ren et al., 2017).

System quality is defined as the general operating performance of an IT system and illustrates whether the system is technically sound (De Lone and McLean, 2003; Gorla et al., 2010; Verma et al., 2018). It is characterized by factors such as reliability, flexibility, response time and adaptability (Gorla et al., 2010; Popovič et al., 2014). In this study, we distinguish between two dimensions of system quality: performance quality and interaction quality. Performance quality (PERQ) is the preliminary operating outcomes such as reliability, flexibility and response time. Interaction quality (INTQ) refers to the advanced operating outcomes such as providing a better user interface to the users, enabling efficient integration of different functions and different BA platforms, and effective adoption to changing contexts (Ji-Fen Ren et al., 2017; Kiron et al.; 2014). INTQ enables linking various users and systems together, either physically or functionally, thus generating organization-wide improvements; however, achieving INTQ is also seen as a challenge by many (Işık et al., 2013).

As businesses increasingly depend on establishing effective BA systems, managers pay more attention to identifying which BA system quality characteristics are required for wide spread use of such systems (Delen and Ram, 2018; Gorla et al., 2010). Although increasing BA adoption rates are reported (Davenpoort and Bean, 2019), there is evidence that even high quality systems do not deliver expected results, casting doubts on the view that there is a link between IS system quality and use (Peters et al., 2016). As a response to the counterintuitive findings, we adopt a contingency perspective and argue that IS quality dimensions need to reflect the needs of different organizational settings, and we propose that analytical decision-making culture $(\mathrm{ADMC})$ is an important boundary condition.

\subsection{Boundary Condition: Analytical Decision-making Culture (ADMC)}

Although the impact of IS quality on the intention to use IT systems is highly acknowledged in previous studies, equivocal findings suggest the need to examine boundary conditions (Busse et al., 2017). A possible contingency is analytical decision-making culture (ADMC) (Popovič et al., 2014), a subset of organizational culture. ADMC is defined as the extent to which a firm encourages decision makers to systematically use data and facts and analyze them for their decision-making tasks (Kulkarni et al., 2017). Previous studies have examined similar concepts, albeit at a more general level, such as organizational support (Lee et al., 2010), top management support (Gangwar et al., 2015) and organizational culture (Shao, 2019) to discuss organizational factors affecting IS adoption. In this study, rather than an overall organizational culture, we specifically focus on the part of organizational culture that supports and fosters adoption of BA tools.

It is argued that in organizations with an ADMC, employees feel encouraged to use data and information, also by adopting advanced statistical and analytical techniques in their decision making process (Popovič et al., 2012; Puklavec et al., 2018). In that sense, ADMC is an enabler making it easy for the users to understand the benefits of BA systems (Cao et al., 2015; Kulkarni et al., 2017). Trieu (2017) proposes that an organizational 
culture favoring analytical, evidence-based decision making has a positive effect on using BA systems. There are also views stating that a strong decision making culture is the key to generating competitive advantage from BA adoption (Božič and Dimovski, 2019).

The main premise in above studies is that in high ADMC environments where employees understand the importance of fact-based decision-making and are encouraged by top management towards using more BA systems, IT solutions will be integrated more in operational and strategic tasks (Grublješič et al., 2015; Popovič et al., 2012). However, not all organizations have high analytical maturity. It is also of interest to understand the factors that makes employees more inclined to use BA systems in low ADMC environments as well. In that respect, we adopt the contingency theory which proposes that context and structure must fit if the organization is to perform well (Burns and Stalker, 1961). Different IS quality dimensions might be equally effective in different organizational contexts; not necessarily requiring a one-size-fits-all approach.

Only a few studies have examined ADMC in relation to BA use, but results have been inconclusive. For instance, Puklavec et al (2018) investigated the direct link between ADMC and business intelligence (BI) evaluation, adoption and use stages, but only found a negative effect to evaluation. Popovič et al. (2012) tested the moderating effect of ADMC on the relationship between content quality, access quality and business intelligence (BI) use, but only found support for the negative effect related to content quality. Sparks and McCann (2015) also tested the same relationships, but they only found a direct effect on BI use. In this study, rather than predicting a moderating effect of ADMC directly on BA use, we predict that the moderating effect is first observed on PEOU and PU. While Popovič et al. (2012) and Sparks and McCann (2015) only focus on INFQ, we also examine two dimensions of system quality - PERQ and INTQ - separately, with the intention to identify more nuanced effects.

\section{Research Model and Hypothesis Development}

This study aims to investigate BA use intentions in organizations by examining individual-, system-, and organizational-level factors. Traditional TAM (Davis, 1989) is adopted to investigate individual-level behavioral concepts whereas it is extended by integrating the IS success model (DeLone and McLean, 1992) to investigate system-level factors. Finally, an important organizational-level determinant, ADMC, is hypothesized as a contingency factor affecting the relationship between the individual- and system-level determinants. All three levels are conceptualized from the perspective of the users.

\subsection{Traditional TAM}

TAM has been validated in several contexts, in both mature and new technology adoptions; therefore, in line with previous research, our first five hypotheses focus on the relationship between core TAM concepts. Similar to previous studies, we hypothesize that a users' intention to use BA systems is positively affected by their attitude toward this technology (Lin and Lin, 2019) (H1). If users have the impression that a BA system is user friendly, clear and understandable, and does not require a lot of mental effort, they will develop a positive attitude about the BA system (Davis, 1989; Vankatesh and Davis, 2000) (H2). Similarly, when a person recognizes the usefulness of descriptive, predictive and prescriptive BA in improving their job performance, this will positively impact their attitude towards BA systems (H3). If users believe that using BA systems will increase their performance at work thus generating a higher level of PU, even if their attitude is not affected, there will be a positive effect on behavioral intentions (Lin and Lin, 2019) (H4). Wu and Wang (2005) argue that PU is a strong determinant, especially in the case of a new technology adoption, which is also in line with our context, recently popular BA use in organizations. Finally, we also hypothesize that PEOU is one of the key determinants of PU (Verma et al., 2018), where improvements in ease of use of also give a sense of usefulness by enabling more work to be done in a shorter period (Brandon-Jones and Kauppi, 2018) (H5). In sum, we formulate the following hypotheses:

$H_{1}$ : There is a positive relationship between attitude to use $B A$ systems and $B A$ use intentions.

$H_{2}$ : There is a positive relationship between PEOU of BA systems and attitude towards BA systems.

$H_{3}$. There is a positive relationship between $P U$ of $B A$ systems and attitude towards $B A$ systems.

$H_{4}$. There is a positive relationship between PU of BA systems and BA use intentions.

$H_{5}$. There is a positive relationship between PEOU of BA systems PU of BA systems. 


\subsection{IS Quality and BA Use Intentions}

IS quality has been argued to improve firm performance by enabling better and more systematic decision making and improving user productivity (Popovič et al., 2014; Verma et al., 2018). Users expect BA systems to provide useful insights and improve efficiency in handling large amount of data; therefore, both information and system quality are prevalent factors affecting user perceptions. Users will be hesitant to use BA systems if the system does not offer valuable information enabling to solve complex problems and is not easy-tounderstand and user-friendly (Ji-fan Ren et al., 2017; Verma et al., 2018). A badly designed BA system not meeting user requirements might even create resistance in the organization, and pose difficulties regarding high quality decision making (Ji-fan Ren et al., 2017; Peters et al., 2016; Popovič et al., 2014). System characteristics impact users' ease and usefulness perceptions (Verma et al., 2018). In highly complex work environments, users might also demand BA systems enabling effective interaction across different users from different departments, possibly also connected by means of related IT systems. Such BA systems offering high INTQ are more likely to be perceived as both easy-to-use and providing valuable outcomes. Thus, the following hypotheses are proposed:

$H_{6}$. There is a positive relationship between INFQ and PEOU of BA systems.

$H 7$. There is a positive relationship between INFQ and PU of BA systems.

$H$ s. There is a positive relationship between PERQ and PEOU of BA systems.

H. There is a positive relationship between PERQ and PU of BA systems.

$H_{10}$. There is a positive relationship between INTQ and PEOU of BA systems.

$H_{11}$. There is a positive relationship between INTQ and PU of BA systems.

\subsection{The Moderating Role of ADMC}

Recent studies suggest that the effect of system quality and information quality on IS use is highly dependent on the context (Popovič et al., 2014). We propose that ADMC is a contingency variable and hence IS quality dimensions have varying effects in low versus high ADMC environments. As the literature is inconclusive, we refrain from making any predictions regarding the direction. In sum, we formulate the following hypothesis:

$H_{12 .}$ ADMC moderates the relationship between system-level and individual-level determinants of $B A$ use intentions.

\subsection{Control variables}

Based on the established stream of IS use literature, we identified one system-level and three individual-level control variables that can affect BA use intentions: system complexity, age, gender and experience (Troilo et al., 2016). Figure 1 illustrates the conceptual model in this study, illustrating the hypotheses and key variables.

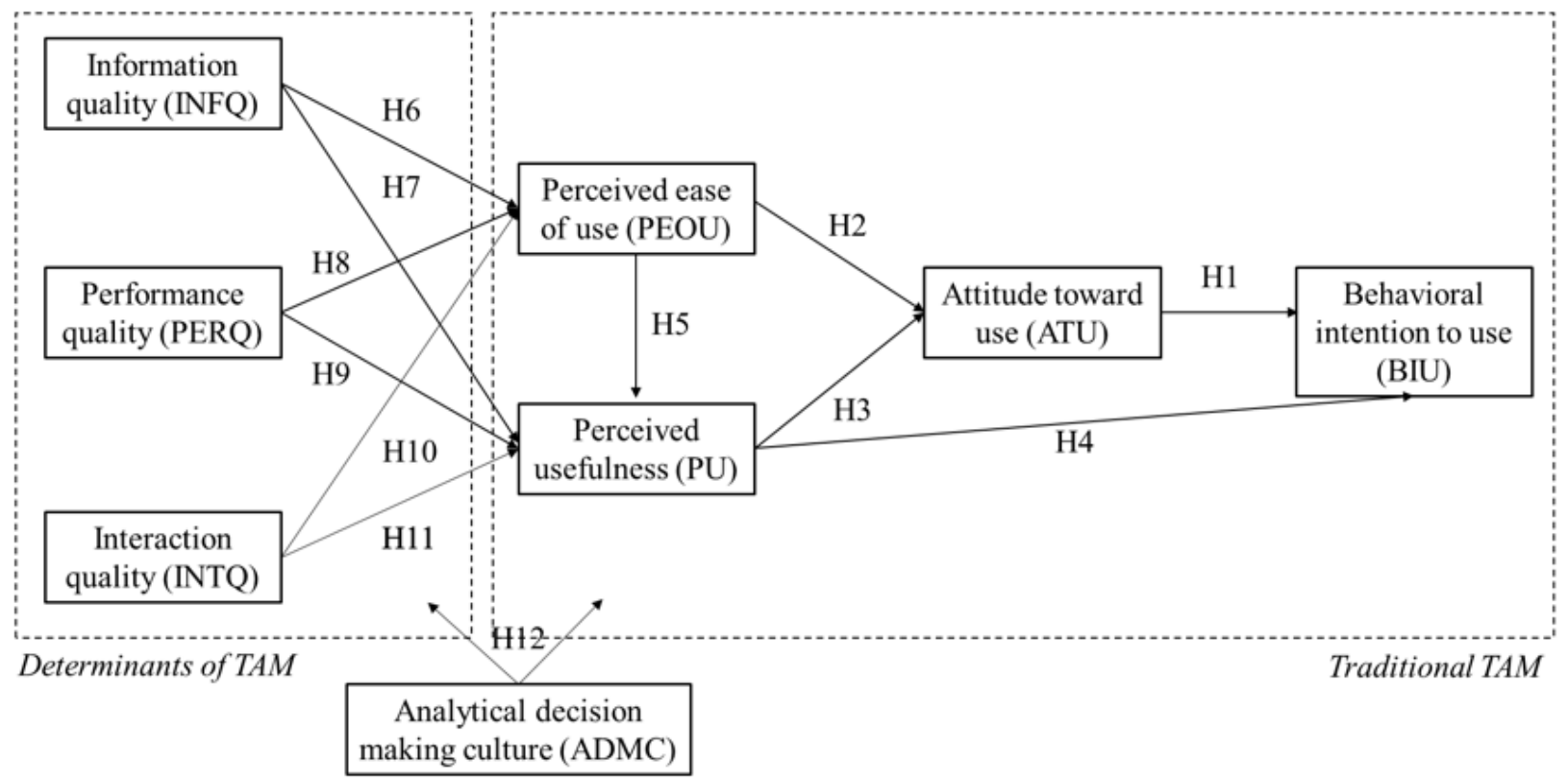

Figure 1. Conceptual model 


\section{Research Method}

\subsection{Data Collection}

We used the survey methodology to test our hypotheses. As BA systems are slowly becoming more popular, we used the purposive sampling method and targeted firms that are known to use BA. For this reason, our sample consists of the key customers of a Turkish BA solutions provider firm. In total, 170 employees of 15 key customers were deemed eligible to participate in this study. In order to increase the response rate, participants were first contacted by phone before sending the invitation email. A reminder e-mail was sent after one and two weeks. In total, 91 employees completed the electronic questionnaire, resulting in a response rate of $53.5 \%$. The sample includes participants from a variety of industries and experience in using BA. Table 1 illustrates the sample descriptives.

Table 1. Sample descriptives

\begin{tabular}{|c|c|c|c|c|c|c|c|c|}
\hline Industry & $n$ & $\%$ & \# Employees & $n$ & $\%$ & Age & $n$ & $\%$ \\
\hline Information tech. & 13 & $14.3 \%$ & $0-50$ & 12 & $13.2 \%$ & $<30$ & 42 & $46.2 \%$ \\
\hline Finance and banking & 18 & $19.8 \%$ & $51-250$ & 16 & $17.6 \%$ & $30-39$ & 36 & $39.6 \%$ \\
\hline Regulatory authority & 18 & $19.8 \%$ & $251-500$ & 5 & $5.5 \%$ & $40-49$ & 11 & $12.1 \%$ \\
\hline Healthcare & 10 & $11.0 \%$ & $501-1000$ & 5 & $5.5 \%$ & $50-59$ & 2 & $2.2 \%$ \\
\hline Chemicals \& petroleum & 11 & $12.1 \%$ & $>1000$ & 53 & $58.2 \%$ & $>59$ & 0 & $0.0 \%$ \\
\hline Fast-moving consumer & 5 & $5.5 \%$ & Total & 91 & $100.0 \%$ & Total & 91 & $100.0 \%$ \\
\hline Energy & 7 & $7.7 \%$ & & & & & & \\
\hline Automotive & 2 & $2.2 \%$ & Education & $n$ & $\%$ & Gender & $n$ & $\%$ \\
\hline Defense & 1 & $1.1 \%$ & High school & 0 & $0.00 \%$ & Female & 45 & $49.5 \%$ \\
\hline Trade & 3 & $3.3 \%$ & University & 65 & $71.40 \%$ & Male & 46 & $50.5 \%$ \\
\hline Service industry & 2 & $2.2 \%$ & Masters & 22 & $24.20 \%$ & Total & 91 & $100.0 \%$ \\
\hline Other & 1 & $1.1 \%$ & Doctorate & 4 & $4.40 \%$ & & & \\
\hline Total & 91 & $100.0 \%$ & Total & 91 & $100 \%$ & & & \\
\hline
\end{tabular}

Table 2. Measurement items

\begin{tabular}{lll}
\hline Constructs & Items & References \\
\hline Analytical & ADMC1. In my organization, I believe that decisions are given & Popovič et al. \\
Decision- & primarily based on rational analysis. & (2012); self- \\
Making & ADMC2. In my organization, the data-based decision-making & constructed \\
Culture & process is well established and known to its stakeholders. &
\end{tabular}

(ADMC) ADMC3. It is my organization's policy to incorporate available information within any decision-making process.

ADMC4. Small or big in any decision making process, we take into account available info.

ADMC5. In my organization, supervisors encourage me to consider situations from all angles.

ADMC6. In my organization, supervisor(s) encourage(s) me to work detailed and methodical.

Attitude $\quad$ ATU1. Using the system is a pleasant experience for me.

toward use ATU2. I feel using the system is a wise choice.

Dulcic et al. (2012);

(ATU)

ATU3. I think that by using the system, we would achieve certain

self-constructed

Behavioral BIU1. I intend to use the system regularly at work.

intention to

BIU2. When I need to do an analysis, I prefer using the system.

Dulcic et al. (2012);

self-constructed

use (BIU)

BIU3. It is likely that I will use the system periodically in the future. 
Information

quality

(INFQ)

Interaction

quality

(INTQ)

Perceived ease

of use (PEU)

Perceived

usefulness

(PU)

Performance

quality

(PERQ)
INFQ1. The system content (the analysis functions presented, etc.) is quite extensive.

INFQ2. The analysis results received from the system is reliable.

INTQ1. The interaction of the system with other operational

systems used in my company is successful.

INTQ2. The system has a user-friendly interface.

PEU1. Learning to use the system was easy for me.

PEU2. Thanks to the system, I can easily do my work.

PEU3. Using the system is clear and understandable.

PEU4. Using the system does not require a lot of mental effort.

PEU5. I do not need a user-manual when using the system.

PEU6. Overall, I find the system easy to use.

PU1. Using the system in my job enables me to accomplish tasks more quickly.

PU2. Using the system improves my job performance.

PU3. The system makes it easier to do my job.

*PU4. The system provides support for important issues at work.

*PU5. Using the system increases my dominance at work.

PU6. Overall, I find the system useful in my job.

PERQ1. The speed of the system is sufficient.

PERQ2. I do not suffer from any data loss in the system and the

system safely stores the entire information.

*PERQ3. The system provides the data in various formats according to the requests.

* removed from scale due to low loadings

\subsection{Measurement}

Majority of the survey questions were adopted from previous research, and new items were formulated if necessary (see Table 2). Question item scales were five-point Likert-type ranging from "1: strongly disagree" to "5: strongly agree". After developing the survey, we first conducted two detailed pre-tests. The first pretest was carried out with the product manager, system consultants, BA system test specialists and software development specialists of the Turkish BA solutions provider firm. The second pretest was conducted with two academicians knowledgeable about the field and the method. As a result, minor modifications were made regarding wording and clarity.

\section{Data Analysis and Results}

We tested our model using partial least squares (PLS), a structural equation modelling (SEM) technique, using the SmartPLS software (version 3.0). Whereas the widely used LISREL and AMOS are covariance-based SEM methods, PLS is a component-based SEM method. We used PLS as it can handle small sample sizes, nonnormal data and complex models with various independent and dependent variables (Chin, 1998; Fornell and Larcker, 1981). PLS relies on a nonparametric bootstrap method, creating subsamples from the original data set until a large number of random subsamples are generated. PLS has two parts: (1) measurement model (to check for measurement reliability and validity) and (2) structural model (relationships between variables).

\subsection{Measurement Model}

Before the structural model can be tested in PLS, we first need to ensure measurement reliability and validity (Chin, 1998; Hulland, 1999). Regarding reliability, we examine item loadings, composite reliabilities, and Cronbach alpha values (Hulland, 1999) (see Table 3). All measurement items have a loading of more than 0.7 (Hulland, 1999). Cronbach alpha ${ }^{1}$ values also indicate high level of measurement reliability, as evidenced by

\footnotetext{
${ }^{1}$ For two-item measures, item correlations are reported.
} 
values higher than 0.7 (Nunnally, 1978). The lowest composite reliability value is 0.847 , which is well above the recommended minimum of 0.7 .

Table 3. Measurement reliability

\begin{tabular}{lllll}
\hline & $\begin{array}{l}\text { Item } \\
\text { loadings }\end{array}$ & $\begin{array}{l}\text { Cronbach's } \\
\text { alpha }\end{array}$ & CR & AVE \\
\hline Analytical decision making culture (ADMC) & N/a & 0.943 & N/a & N/a \\
Attitude toward use (ATU) & $0.867-0.919$ & 0.867 & 0.919 & 0.790 \\
Behavioral intention to use (BIU) & $0.922-0.961$ & 0.931 & 0.956 & 0.879 \\
Information quality (INFQ) & $0.823-0.891$ & $0.475^{* *_{a}}$ & 0.847 & 0.736 \\
Integration quality (INTQ) & $0.871-0.898$ & $0.570^{* *_{a}}$ & 0.878 & 0.783 \\
Perceived ease of use (PEOU) & $0.774-0.890$ & 0.857 & 0.902 & 0.698 \\
Perceived usefulness (PU) & $0.807-0.915$ & 0.930 & 0.945 & 0.742 \\
Performance quality (PERQ) & $0.795-0.806$ & $0.563^{* *_{a}}$ & 0.878 & 0.783 \\
\hline
\end{tabular}

a: Inter-item correlations, ${ }^{* *} \mathrm{p}<0.01$

Regarding construct validity, two elements have been examined: convergent validity and discriminant validity (Gefen and Straub, 2005) (see Table 4). Convergent validity is achieved as the lowest average variance extracted (AVE) for our constructs is 0.698, exceeding the recommended minimum of 0.5 (Chin, 1998). Discriminant validity is achieved as the square roots of the AVEs are greater than the correlations with all other constructs (See Table 4).

Table 4. Discriminant validity

\begin{tabular}{lccccccc}
\hline & (ATU) & (BIU) & (INFQ) & (INTQ) & (PEOU) & (PU) & (PERQ) \\
\hline Attitude towards use (ATU) & $\mathbf{0 . 8 8 9}$ & & & & & & \\
Behavioral intention to use (BIU) & 0.847 & $\mathbf{0 . 9 3 8}$ & & & & & \\
Information quality (INFQ) & 0.623 & 0.552 & $\mathbf{0 . 8 5 8}$ & & & & \\
Integration quality (INTQ) & 0.553 & 0.512 & 0.379 & $\mathbf{0 . 8 8 5}$ & & & \\
Perceived ease of use (PEOU) & 0.620 & 0.712 & 0.258 & 0.644 & $\mathbf{0 . 8 3 6}$ & & \\
Perceived usefulness (PU) & 0.832 & 0.834 & 0.590 & 0.599 & 0.666 & $\mathbf{0 . 8 6 1}$ & \\
Performance quality (PERQ) & 0.626 & 0.660 & 0.761 & 0.400 & 0.403 & 0.671 & $\mathbf{0 . 8 0 1}$ \\
\hline
\end{tabular}

Common method bias is a systematic error that is caused by the measurement method used in the study (Kock, 2015). It can pose a threat especially in survey research when all variables are perceptual and/or data is collected from a single respondent. To check for common method bias, we conducted Harman's (1960) single factor test to see if a single factor constitutes the majority of the covariance between variables (Podsakoff et al., 2003). Based on exploratory principal components factor analysis, we find that $43 \%$ of the variance is explained by the first factor, which is below the commonly accepted threshold of 50\% (Harman, 1960). Therefore, it can be concluded that this study does not suffer from common method bias. 


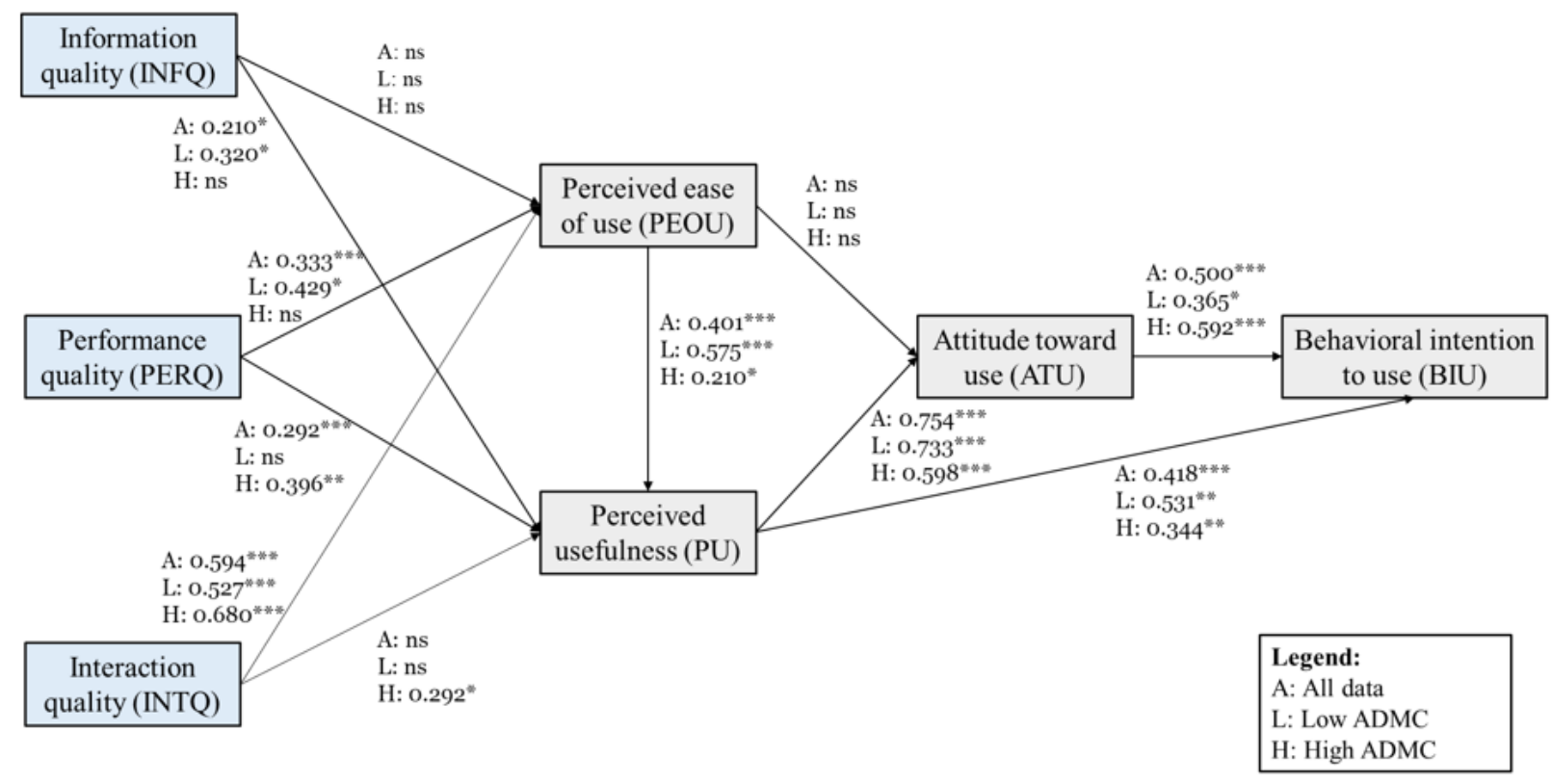

Figure 2. Summary of findings

\subsection{Structural Model}

Attitude to use BA (ATU) was found to be positively associated with the intention to use BA (BIU) $(\Upsilon=0.500$, $\mathrm{p}=0.000)$, supporting H1. However, PEOU did not have a significant effect on ATU $(\Upsilon=0.117, n s)$, thus rejecting H2. PU was found to be positively associated with both ATU $(\Upsilon=0.754, \mathrm{p}=0.000)$ and BIU $(\Upsilon=0.418, \mathrm{p}=0.000)$, supporting $H 3$ and $H 4$. We found that a higher level of PEOU leads to PU $(\Upsilon=0.401, p=0.000)$ supporting $H 5$. Combining the findings about $\mathrm{H} 2$ and $\mathrm{H} 5$, we also checked for the indirect effect of PEOU on ATU via PU. We found this effect to be positive and significant $(Y=0.303, p=0.000)$, highlighting that rather than a direct effect, PEOU has an indirect effect on ATU. Overall, the results illustrate that TAM successfully explains BA use intentions.

When the antecedents of TAM are examined, we find that information quality (INFQ) does not have an impact on PEOU $(\Upsilon=-0.220, n s)$, rejecting H6. However, in line with previous findings we find that INFQ increases PU $(\Upsilon=0.210, p=0.05)$, supporting $H 7$. We conceptualize system quality in two dimensions: performance quality (PERQ) and interaction quality (INTQ). We find that PERQ has a positive impact on both PEOU ( $Y=$ 0.333, $\mathrm{p}=0.01)$ and $\mathrm{PU}(\Upsilon=0.292, \mathrm{p}=0.001)$, supporting H8 and H9. INTQ, on the other hand, was found to be associated with a higher level of PEOU $(\Upsilon=0.594, \mathrm{p}=0.000)$, supporting H10, but was not related to PU $(\Upsilon=$ $0.144, n s)$, rejecting $H 11$.

To evaluate the predictive power of our model, we checked the explained variance $\left(R^{2}\right)$ of the endogenous variables and overall fit statistics (Peng and Lai, 2012) (See Table 5). We found that $\mathrm{R}^{2}$ values are moderate to substantial $\left(R^{2}{ }_{B I U}=0.767, R^{2}{ }_{A T U}=0.693, R^{2}{ }_{\mathrm{PU}}=0.658, R^{2}{ }_{\mathrm{PEU}}=0.442\right)$ (Peng and Lai, 2012). We checked the model fit using the goodness-of-fit (GoF) formula (Tenenhaus et al., 2005). For our structural model, GoF value is 0.694, quite above the accepted cut-off value of 0.36 (Fornell and Larcker, 1981). 
N. Bayram - M. Akın Ateş 12/1 (2020) 583-598

Table 5. Structural model and findings

\begin{tabular}{|c|c|c|c|c|c|c|c|c|}
\hline & \multicolumn{6}{|c|}{ Path coefficients and significance } & \multirow{2}{*}{\multicolumn{2}{|c|}{$\begin{array}{l}\text { Coefficient difference } \\
\text { test }\end{array}$}} \\
\hline & \multicolumn{2}{|c|}{$\begin{array}{l}\text { ALL DATA } \\
(\mathrm{n}=91)\end{array}$} & \multicolumn{2}{|c|}{$\begin{array}{l}\text { High ADMC } \\
(\mathrm{n}=51)\end{array}$} & \multicolumn{2}{|c|}{$\begin{array}{l}\text { Low ADMC } \\
(\mathrm{n}=40)\end{array}$} & & \\
\hline & path c. & $\mathrm{p}$ & path c. & $\mathrm{p}$ & path c. & $\mathrm{p}$ & PLS-MGA & Parametric \\
\hline H1: ATU $\rightarrow$ BIU & 0.500 & 0.000 & 0.569 & 0.000 & 0.365 & 0.033 & 0.156 & 0.299 \\
\hline H2: PEOU $\rightarrow$ ATU & 0.117 & 0.236 & 0.084 & 0.504 & 0.182 & 0.353 & 0.640 & 0.659 \\
\hline H3: PU $\rightarrow$ ATU & 0.754 & 0.000 & 0.598 & 0.000 & 0.733 & 0.000 & 0.736 & 0.475 \\
\hline H4: PU $\rightarrow$ BIU & 0.418 & 0.000 & 0.344 & 0.006 & 0.531 & 0.001 & 0.821 & 0.351 \\
\hline H5: PEOU $\rightarrow$ PU & 0.401 & 0.000 & 0.210 & 0.085 & 0.575 & 0.000 & 0.983 & 0.034 \\
\hline H6: INFQ $\rightarrow$ PEOU & -0.220 & 0.088 & -0.233 & 0.322 & -0.095 & 0.671 & 0.668 & 0.674 \\
\hline H7: INFQ $\rightarrow$ PU & 0.210 & 0.034 & 0.041 & 0.802 & 0.320 & 0.080 & 0.889 & 0.252 \\
\hline H8: PERQ $\rightarrow$ PEOU & 0.333 & 0.008 & 0.086 & 0.637 & 0.429 & 0.029 & 0.901 & 0.201 \\
\hline H9: PERQ $\rightarrow$ PU & 0.292 & 0.001 & 0.396 & 0.003 & 0.135 & 0.415 & 0.092 & 0.208 \\
\hline H10: INTQ $\rightarrow$ PEOU & 0.594 & 0.000 & 0.680 & 0.000 & 0.527 & 0.000 & 0.189 & 0.372 \\
\hline H11: INTQ $\rightarrow$ PU & 0.144 & 0.113 & 0.292 & 0.016 & -0.002 & 0.986 & 0.054 & 0.112 \\
\hline
\end{tabular}

\subsection{Moderation Analysis}

To test for the moderating effect of ADMC, we performed a multi-group analysis (PLS-MGA) and permutation in PLS (Hair et al., 2018). Based on the mean of ADMC, we divided the data set into two parts as high ADMC $(n=51)$ and low ADMC $(n=40)$. The results suggest that ADMC does not have an impact on the main TAM relationships (H1-H5), but it acts as a moderator on the determinants of TAM (H6-H11) (see Table 5).

We find that INFQ has a positive impact on PU only in low ADMC group $(\Upsilon=0.320, p=0.05)$, but has no effect in high ADMC group $(\Upsilon=0.041, n s)$. Similarly, PERQ has a positive impact on PEU $(\Upsilon=0.429, \mathrm{p}=0.05)$ in low ADMC group, but no effect in high ADMC group $(\Upsilon=0.086, n s)$. Interestingly, PERQ has a positive impact on PU in high ADMC group $(\Upsilon=0.396, p=0.01)$, but no effect in low ADMC group $(\Upsilon=0.135, n s)$. We were not able to find support for the positive association between INTQ and PU for the whole data; but moderation analysis suggests that INTQ has a positive impact in high ADMC group $(\Upsilon=0.292, \mathrm{p}=0.05)$, but not in low ADMC group $(\Upsilon=-0.002, n s)$. ADMC does not have an impact on the relationship between INFQ and PEOU; in both groups, path coefficients are not significant. Similarly, ADMC does not have an impact on the relationship between INTQ and PEOU; in both groups, there is a positive effect, yet the difference between path coefficients is not significant. Therefore, our results provide partial support for the moderating role of ADMC.

\section{Discussion and Conclusion}

\subsection{Theoretical Implications}

In response to the calls for more research on user acceptance of BA systems (Jaklič et al., 2018; Kiron et al., 2014), we developed an integrative research model that combines two well-known theoretical models, namely TAM and IS success models, and extended them by examining an important organizational contingency, namely ADMC. Our study makes an important theoretical contribution to the research on technology acceptance (Davis, 1989) in general and to BA acceptance literature (Verma et al., 2018) in particular. Specifically, our results suggest that the extended TAM with system-level antecedents in light of an organizational-level contingency (i.e. ADMC) could better explain employee's intention to use BA systems.

We find strong support for the widely accepted traditional TAM (Davis et al., 1989). First, we find that a positive attitude toward using the BA tools leads to a higher level of behavioral intention to use these tools. Attitudes represent an individual's tendency to feel, think or behave in a positive or negative direction (Vakola and Nikolaou, 2005). Attitude affects analytical thinking of the individual, and employees who have a positive attitude towards using the system are more likely to have intention to use the business analytics (Mansell, 2015). Literature suggest two key determinants of ATU: PEU and PU. We find support for a strong effect ATU on PU, but we only find an indirect effect of PEU on ATT, via PU. Thus, our findings are in line with the view that the main determinant of ATU is PU, and PEU first causes an increase in PU, which in the end increases ATU. 
Previous studies investigating the link between INFQ and PEU provide inconsistent findings; some find a positive association (e.g. Çelik and Yllmaz, 2011) and some find no effect (e.g. Kim et al., 2008). Our results support the latter view; we found that INFQ does not have an impact on PEU. On the other hand, previous studies find a stronger support in general for the link between INFQ and PU (Calisir et al., 2014; Kim et al., 2008; Lin, 2010). We also find a positive association between INFQ and PU; however, only in low ADMC environment. This might seem contradictory in the first place; however, it can also be interpreted that users in high ADMC environments expect INFQ as a necessary condition that has to be present in any BA system; therefore, not see it as a differentiating factor increasing PU.

System quality is often argued to be a factor affecting whether users perceive an IS system as easy or not (Calisir et al., 2014). In this study, we distinguish two dimensions of system quality: INTQ and PERQ. As expected, we find that INTQ has a positive impact on PEU, but PERQ has an impact on PEOU only in low ADMC environments. Previous studies also support the view that system quality increases the likelihood that an IS system will be perceived as useful (Calisir et al., 2014, Kim et al., 2008). Interestingly, we found that both dimensions of system quality have a positive impact on PU only in high ADMC environments. Combining these findings, one can argue that users in low ADMC environments think their job becomes easier due to high system quality, yet they fail to see the connection to increased value. Contrarily, in high ADMC environments, users only see INTQ as a factor affecting PEOU, yet they think both INTQ and PERQ adds value to their daily tasks and operations by means of better user interface, compatibility with other systems, and reliable data storage.

In line of the above discussions, we find partial support for the moderating role of ADMC, which confirms with the view that decision-makers' choice of using information and analytics is highly influenced by the presence of ADMC in the organization (Popovič et al., 2012). In that regard, we are among the first to illustrate that certain IS quality characteristics can motivate users to adopt BA systems in low ADMC environments as well, as opposed to the commonly held view that high ADMC is better for BA adoption.

\subsection{Managerial Implications}

Our research carries important insights for both managers and BA system providers. As our results suggest, ADMC has a huge effect on whether certain BA system quality characteristics generate favorable user perceptions or not, via diverging effects on PEOU and PU. For instance, in low ADMC environments, INFQ can be seen as a "game-changer", as it improves PU, which is the most important predictor of BA use intentions. In contrast, PERQ and INTQ are "nice-to-have" factors, as they only increase PEOU, which only has an indirect effect via PU. In low ADMC environments, users are not in the habit of integrating fact-based decision making in their daily tasks and there are fragmented approaches to using data. In such a case, users are astonished when they realize the benefits they can achieve from high INFQ offering extensive information in a reliable way. In contrast, in high ADMC environments, users are encouraged by their supervisors to incorporate available information within any decision-making process. It is interesting to note that in such environments, INFQ is not a distinguishing factor affecting perceptions of ease or usefulness; instead, it is "taken-for-granted"; otherwise, users would not adopt such BA systems in the first place. In high ADMC environments, PERQ is regarded as a "user-friendly" factor, improving ease perceptions, whereas INTQ becomes the "game-changer" by favorably impacting both ease and usefulness perceptions of users. Combining these findings, one can argue that when designing BA systems, BA system providers should prioritize INFQ for low ADMC environments, but acknowledge that in high ADMC environments this would not suffice. Instead, in such environments they should highlight more the PERQ, and more importantly, INTQ enabling users and functions to interact with each other, offering more integrated solutions to facilitate the BA adoption process.

\subsection{Limitations and Suggestions for Future Research}

Similar to any other study, this research is not without limitations. First, regarding the boundary conditions, we only focus on the contingency effect of a single organizational dimension; i.e. ADMC. Future studies can investigate other organizational factors and aim to tackle the complex interplay between them in relation to BA adoption. Second, due to the cross-sectional nature of the study, we cannot make causal inferences. Future studies can also examine BA use intentions over time in longitudinal data collection efforts or with experimental settings. Third, although our sample presents a good variety in terms of industries, the relatively 
small sample size and the focus on a single, developing country context limits the generalizability of the findings. Against this shortcoming, we encourage researchers to test and extend our model in larger, multicountry samples to advance the understanding about BA use intentions across different cultures. Finally, future research can aim for measuring actual BA use rather than intentions by using objective data, for instance examining BA system logs (e.g. hours spent, modules of BA used, etc.).

Notwithstanding the limitations discussed above, we believe that our findings would aid future studies investigating boundary conditions regarding BA use and examining alternative approaches for increasing BA adoption in different organizational contexts in line with users' changing needs and requirements.

\section{REFERENCES}

Alhirz, S. (2015). "Do cultural dimensions differentiate ERP acceptance? A study in the context of Saudi Arabia", Information Technology \& People, 28 (1), 163-194.

Alomari, M., Woods, P. and Sandhu, K. (2012). “Predictors for e-government adoption in Jordan: Deployment of an empirical evaluation based on a citizen-centric approach", Information Technology $\&$ People, 25 (2), 207-234.

Arnott, D., Lizama, F. and Song, Y. (2017). "Patterns of business intelligence systems use in organizations", Decision Support Systems, 97 (May), 58-68.

Bach, M.P., Čeljo, A. and Zoroja, J. (2016). “Technology acceptance model for business intelligence systems: Preliminary research", Procedia Computer Science, 100, 995-1001.

Božič, K. and Dimovski, V. (2019). "Business intelligence and analytics for value creation: The role of absorptive capacity", International Journal of Information Management, 46 (June), 93-103.

Busse, C., Kach, A.P. and Wagner, S.M. (2017). “Boundary conditions: What they are, how to explore them, why we need them, and when to consider them", Organizational Research Methods, 20 (4), 574-609.

Brandon-Jones, A. and Kauppi, K. (2018). "Examining the antecedents of the technology acceptance model within e-procurement", International Journal of Operations \& Production Management, 38 (1), 22-42.

Burns, T. and Stalker, G. M. (1961). “The Management of Innovation”, London, Tavistock.

Calisir, F., Altin Gumussoy, C., Bayraktaroglu, A.E. and Karaali, D. (2014). "Predicting the intention to use a web-based learning system: perceived content quality, anxiety, perceived system quality, image, and the technology acceptance model", Human Factors and Ergonomics in Manufacturing $\mathcal{E}$ Service Industries, 24 (5), 515-531.

Cao, G. and Duan, Y. (2017). "How do top- and bottom-performing companies differ in using business analytics?", Journal of Enterprise Information Management, 30 (6), 874-892.

Chin, W.W. (1998). “The Partial Least Squares Approach for Structural Equation Modelling," in: G.A. Marcoulides (Ed.), Modern Methods for Business Research, London, Lawrance Erlbaum Associates, 295336.

Côrte-Real, N., Ruivo, P., Oliveira, T. and Popovič, A. (2019). “Unlocking the drivers of big data analytics value in firms", Journal of Business Research, 97 (April), 160-173.

Çelik, H.E. and Yilmaz, V. (2011). "Extending the technology acceptance model for adoption of e-shopping by consumers in Turkey", Journal of Electronic Commerce Research, 12(2), 152-164.

Daradkeh, M.K. (2018). “Determinants of visual analytics adoption in organizations: Knowledge discovery through content analysis of online evaluation reviews", Information Technology \& People, 32 (3), 668695.

Davenport, T.H. and Bean, R. (2019). "Big data and AI executive survey 2019 summary of findings", available at: https://newvantage.com/wp-content/uploads/2018/12/Big-Data-Executive-Survey-2019-FindingsUpdated-010219-1.pdf (accessed 13 June 2019). 


\section{N. Bayram - M. Akın Ateş 12/1 (2020) 583-598}

Davenport, T.H. and Harris, J.G., (2007). "Competing on Analytics: The New Science of Winning", Harvard Business School Press, Brighton, Boston.

Davis, F. D. (1989). "Perceived usefulness, perceived ease of use, and user acceptance of information technology", MIS Quarterly, 13 (3), .319-340.

Delen, D. and Ram, S. (2018). "Research challenges and opportunities in business analytics", Journal of Business Analytics, 1 (1), .2-12.

DeLone, W.H. and McLean, E.R. (2003). "The DeLone and McLean model of information systems success: a ten-year update", Journal of Management Information Systems, 19 (4), .9-30.

Fishbein, M. and Ajzen, I. (1975). "Belief, Attitude, Intentions and Behavior: An Introduction to Theory and Research", Addison-Wesley, Reading.

Fornell, C.R. and Larcker, D.F. (1981). "Structural equation models with unobservable variables and measurement error", Journal of Marketing Research, 18 (3), 39-50.

Gangwar, H., Date, H. and Ramaswamy, R. (2015). "Understanding determinants of cloud computing adoption using an integrated TAM-TOE model", Journal of Enterprise Information Management, 28 (1), 107-130.

Gefen, D. and Straub, D.W. (2005). "A practical guide to factorial validity using PLS-graph: Tutorial and annotated example", Communications of the Association for Information Systems, 16 (5), 91-109.

Gorla, N., Somers, T. M. and Wong, B. (2010). “Organizational impact of system quality, information quality: and service quality", The Journal of Strategic Information Systems, 19 (3), 207-228.

Grublješič, T., Coelho, P.S. and Jaklič, J. (2015). "Antecedent determinants of beliefs underpinning the intention to accept and use business intelligence systems: The importance of organizational factors", Issues in Information Systems, 16 (4), 166-177.

Hair, J.F., Sarstedt, M., Ringle, C.M. and Gudergan, S.P. (2018). "Advanced Issues in Partial Least Squares Structural Equation Modeling (PLS-SEM)", Sage, Thousand Oaks, CA.

Harman, H.H. (1960). "Modern Factor Analysis", University of Chicago Press, Chicago:IL.

Hong, S., Lui, C.S.M., Hahn, J., Moon, J.Y. and Kim, T.G. (2013). “How old are you really? Cognitive age in technology acceptance", Decision Support Systems, 56, 122-130.

Hulland, J. (1999). “Use of partial least squares (PLS) in strategic management research: A review of four recent studies", Strategic Management Journal, 20 (2), 195-204.

Işık, Ö., Jones, M.C. and Sidorova, A. (2013). "Business intelligence success: The roles of BI capabilities and decision environments", Information \& Management, 50 (1), 13-23.

Jaklič, J., Grublješič, T. and Popovič, A. (2018). "The role of compatibility in predicting business intelligence and analytics use intentions", International Journal of Information Management, 43 (December), 305-318.

Ji-fan Ren, S., Fosso Wamba, S., Akter, S., Dubey, R. and Childe, S.J. (2017). “Modelling quality dynamics, business value and firm performance in a big data analytics environment", International Journal of Production Research, 55 (17), 1-16.

Kim, T.G., Lee, J.H. and Law, R. (2008). "An empirical examination of the acceptance behaviour of hotel front office systems: An extended technology acceptance model”. Tourism Management, 29 (3), 500-513..

Kiron, D., Prentice, P.K. and Ferguson, R.B. (2014). "The analytics mandate”, MIT Sloan Management Review, 55 (January), 1-25.

Kock, N. (2015). "Common method bias in PLS-SEM: A full collinearity assessment approach", International Journal of e-Collaboration, 11 (4), .1-10.

Kulkarni, U., Robles-Flores, J.A. and Popovič, A. (2017). "Business intelligence capability: the effect of top management and the mediating roles of user participation and analytical decision-making orientation", Journal of the Association for Information Systems, 18 (7), 516-541. 
Lee, D.H., Lee, S.M., Olson, D.L. and Chung, S.H. (2010). "The effect of organizational support on ERP implementation", Industrial Management and Data Systems, 110 (2), 269-283.

Lin, C. and Lin, M. (2019). "The determinants of using cloud supply chain adoption", Industrial Management $\mathcal{E}$ Data Systems, 119 (2), 351-366.

Lin, F., Fofanah, S.S. and Liang, D. (2011). “Assessing citizen adoption of e-Government initiatives in Gambia: A validation of the technology acceptance model in information systems success", Government Information Quarterly, 28 (2), 271-279.

Naicker, V. and van der Merwe, D.B. (2017). “Managers' perception of mobile technology adoption in the Life Insurance industry", Information Technology \& People, 31 (2), 507-526.

Nam, D., Lee, J. and Lee, H. (2019). “Business analytics use in CRM: A nomological net from IT competence to CRM performance", International Journal of Information Management, 45 (April), 233-245.

Nunnally, J.C. (1978). "Psychometric Theory", McGraw-Hill, New York.

Podsakoff, P., MacKenzie, S., Lee, J. and Podsakoff, N. (2003). "Common method biases in behavioural research: A critical review of the literature and recommended remedies," Journal of Applied Psychology, 88 (5), 879-903.

Peng, D.X. and Lai, F. (2012). “Using partial least squares in operations management research: A practical guideline and summary of past research", Journal of Operations Management, 30 (6), 467-480.

Peters, T., Işık, Ö., Tona, O. and Popovič, A. (2016). "How system quality influences mobile BI use: The mediating role of engagement", International Journal of Information Management, 36 (5), 773-783.

Popovič, A., Hackney, R., Coelho, P.S. and Jaklič, J. (2012). “Towards business intelligence systems success: Effects of maturity and culture on analytical decision making", Decision Support Systems, 54 (1), 729739.

Popovič, A., Hackney, R., Coelho, P.S. and Jaklič, J. (2014). “How information-sharing values influence the use of information systems: an investigation in the business intelligence systems context", The Journal of Strategic Information Systems, 23 (4), 270-283.

Popovič, A., Hackney, R., Tassbehji, R. and Castelli, M. (2018). “The impact of big data analytics on firms' high value business performance", Information Systems Frontiers, 20 (2), 209-222.

Puklavec, B., Oliveira, T. and Popovič, A. (2018). "Understanding the determinants of business intelligence system adoption stages: An empirical study of SMEs", Industrial Management \& Data Systems, 118 (1), 236-261.

Raguseo, E. and Vitari, C. (2018). "Investments in big data analytics and firm performance: An empirical investigation of direct and mediating effects", International Journal of Production Research, 56 (15), 52065221.

Rauniar, R., Rawski, G., Yang, J. and Johnson, B. (2014). "Technology acceptance model (TAM) and social media usage: an empirical study on Facebook", Journal of Enterprise Information Management, 27 (1), 630.

Shao, Z. (2019). "Interaction effect of strategic leadership behaviors and organizational culture on IS-business strategic alignment and enterprise systems assimilation", International Journal of Information Management, 44 (February), 96-108.

Sparks, B.H. and McCann, J.T. (2015). “Factors influencing business intelligence system use in decision making and organisational performance", International Journal of Sustainable Strategic Management, 5 (1), 31-54.

Tenenhaus, M., Vinzi, V.E., Chatelin, Y.M. and Lauro, C. (2005). "PLS path modelling”, Computational Statistics and Data Analysis, 48 (1), 159-205.

Trieu, V.H. (2017). “Getting value from business intelligence systems: A review and research agenda”, Decision Support Systems, 93, 111-124. 
N. Bayram - M. Akın Ateş 12/1 (2020) 583-598

Troilo, M., Bouchet, A., Urban, T.L. and Sutton, W.A (2016). "Perception, reality, and the adoption of business analytics: Evidence from North American professional sport organizations", Omega, 59, 72-83.

Verma, S., Bhattacharyya, S.S. and Kumar, S. (2018). "An extension of the technology acceptance model in the big data analytics system implementation environment", Information Processing and Management, 54 (5), 791-806.

Wixom, B.H. and Todd, P.A. (2005). "A theoretical integration of user satisfaction and technology acceptance", Information Systems Research, 16, 85-102.

$\mathrm{Wu}$, J.H. and Wang, S.C. (2005). "What drives mobile commerce? An empirical evaluation of the revised technology acceptance model", Information and Management, 42 (5), 719-729.

Yeoh, W. and Popovič, A. (2016). "Extending the understanding of critical success factors for implementing business intelligence systems", Journal of the Association for Information Science and Technology, 67 (1), 134-147. 